\title{
Opportunistic Offloading Scheme in Heterogeneous Vehicular Network
}

\author{
Mahmoud Alawi, Elankovan Sundararajan, Abdullah Mohd. Zin, Raed Alsaqour, Mahamod \\ Ismail
}

\begin{abstract}
A Heterogeneous Vehicular Network (HetVNET) is a promising network system that integrates several network protocols or systems e.g. IEEE802.11p, Short Range Communication, Third/Fourth Generation mobile networks etc. In HetVNET, wireless fidelity access points (Wi-Fi APs) must utilized by automobile end users to stabilise the Long-Term Evolution (LTE) $4 G$ networks by way of offloading. No matter how usage of the opportunistic Wi-Fi APs to offload the LTE networks in a HetVNET system is comparatively more straightforward. This circumstances is due to the less area reached by Wi-Fi access points and weak placement strategies of access points. Many researcher have suggested that offloading schemes based on the historical wireless fidelity connection patterns noticed by an interest vehicle (IV) in making an offloading conclusion. However, based only on the historical connection patterns affects the prediction accuracy and offloading ratio of most existing schemes even when access point placement data is obtainable. This work suggested that the Opportunistic Offloading Scheme (OOS) is based on historical connection patterns and vehicular trajectory computation to predict the next accessible access point. The suggested plan is decentralised and focuses on urban scenarios whereby the likelihood of an IVto come into contact with Wi-Fi access points is high. We detect the complexity of the suggested Opportunistic Offloading Scheme in this work. The result shows that Opportunistic Offloading Scheme is linear dependent on the amount of the processed data accessible for the prediction.
\end{abstract}

Keywords:- Opportunistic offloading, Heterogeneous Vehicular Network, Vehicular Communication, Markov predictor. Wi-Fi, LTE

\section{INTRODUCTION}

Of late, we notice an increase in mobile data gadget with an increasing number of data-hungry applications, which demand unlimited Internet services. Cisco's latest projection shows that by the end of 2021, there will 1.5 mobile gadget per capita, and the total number of mobile gadgets will approximately 11.6 billion [1]. These gadgets will need a direct connection to mobile networks. Given this expectation, we conclude that there will be cellular network overload shortly. Several studies have preferred wireless fidelity (Wi-Fi) or Femtocell as alternative networks to offload mobile traffic [2,3]. However, Wi-Fi is already

Revised Manuscript Received on April 12, 2019.

Mahmoud Alawi, Research Center for Software Technology and Management Faculty of Information and Technology Universiti Kebangsaan Malaysia, 43600, Bangi Selangor, Malaysia. (mahmoud@siswa, elan@\}ukm.edu.my)

Elankovan Sundararajan Abdullah Mohd. Zin, Research Center for Software Technology and Management Faculty of Information and Technology Universiti Kebangsaan Malaysia, 43600, Bangi Selangor, Malaysia.

Raed Alsaqour, Department of Computer Science, College of Computing and Informatics, Saudi Electronic University, 2360, Jeddah, Saudi Arabia. (raed@ukm.edu.my)

Mahamod Ismail Department of Electrical, Electronic and System Engineering, Universiti Kebangsaan Malaysia,43600, Bangi Selangor Malaysia. (mahamod@ukm.edu.my) widely utilized as a standard offloading medium [4]. Several traffic parts directed to the cellular network are re-directed to the Wi-Fi network. This methodology can lower the congestion in mobile networks and the cost imposed on the network users. People generally spend a considerable time on the either highway or roadway and require a suitable connection to the Internet or another automobile network [5]. The automotive industry and research community are contemplating installing networks among vehicles to enable inter-vehicle communication utilizing ad hoc architecture, which is also known as vehicular ad-hoc network (VANET). However, VANET itself cannot cope with the increasing demands of network applications that vehicle users require while travelling. There is a possibility of integration of VANET with other network technologies, such as WiMAX, Wi-Fi, and cellular networks[i.e., third generation (3G), 4G long-term evolution (LTE), and 4GLTE advanced (LTE-A)] to form a robust and reliable HetVNET[6]. This particular cellular network is the best option in providing pervasive and dependable Internet access to vehicles because it is well deployed and covers larger areas compared with other types of network technologies [7]. However, the simple integration of VANET to mobile networks can elevate the overload problem and degrade the QoS of both vehicle and non-vehicle users [8].

The current study recommended an OOS in HetVNET architecture. The motive of the recommended opportunistic offloading scheme (OOS) methodology is to provide vehicle users prior processed data of the next Wi-Fi availability and its expected data capacity in making proactive offloading conclusions. The recommended opportunistic offloading scheme (OOS) methodology utilizes three criteria in predicting the future Wi-Fi AP availability and its expected capacity: historical connection patterns, vehicle trajectory, and historical data rate information. The recommended OOS methodology employs historical connection patterns and vehicle trajectory computation, as well as foresees the next accessible Wi-Fi AP. Moreover, it utilizes the historical data rate observed by the interesting automobile to predict the average data rate used by said IV, which helps optimise the offloading conclusion. The offloading conclusion is based on the delay requisite of the applications together with the expected capacity of the future Wi-Fi AP.

We organise this paper as follows. Section 2 concisely surveyed related works. The section 3 explains the recommended OOS. The section 4 discusses the performance complexity of the recommended OOS. Finally, Section 5 presents the conclusions.

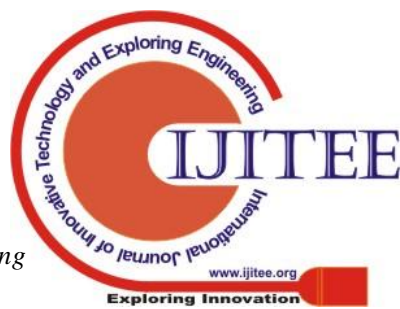




\section{RELATED WORK}

Recently, several recommended Vehicular Wi-Fi offloading methods are accessible in this domain. The objective of these methodology is to employ Wi-Fi access points come into contact with the automobiles to offload mobile data. However, the automobile must have prior processed data about the availability of the next access points and their expected data capacity for the recommended method to be feasible. This methodology can help the automobile user make an earlier conclusion depending on the delay application requisite imposed by the particular application.

Lee and Lee [9] recommended offloading techniques depends on a historical table supported and updated by every moving automobile to upgrade the offloading efficiency and prediction error. The historical connection table accumulates the coordinates of the access points accessed by the automobiles alongside the either highway or roadway. Their work defines on the urban scenario, and a GPS is treated to be convenient for obtaining the automobile placements. The historical prediction table in [9] accumulates only the present and consequent access points come into contacted. Moreover, the prediction process only starts when the table has sufficient data for prediction. Two circumstances must be evaluated and satisfied with the automobile to make an offloading conclusion:

- The approximated time to reach to the next access points must be less than the delay requisite of the particular application,

- The expected data capability of the following access point must be larger than the predicted amount the automobile would send to the next access points.

When both circumstances are satisfied, the automobile will wait and start the transfer only upon come into contacting the next access point. The offloading efficiency at a speed of $10 \mathrm{~m} / \mathrm{s}$ is lower than that of high-speed automobiles (i.e., $15 \mathrm{~m} / \mathrm{s}$ ). The ineffectiveness of the present access point to anticipate the future access point is due to the automobile utilising an alternate route or coming from an opposite direction, which results in a potential undiscovered future access point.

Balasubramanian et al. [10] recommended the Wiffler model to relieve the $3 \mathrm{G}$ capacity. This model predicts the next Wi-Fi connectivity utilising the historical number of Wi-Fi access point come into contacted. The recommended model makes the offloading conclusion based on the maximum delay requisite of several applications. It checks if the delay requisite is not tight and the transmission to $\mathrm{Wi}-\mathrm{Fi}$ is completed within the application delay threshold. The data will wait and transfer only when we come into contact Wi-Fi access point. Otherwise, the data is transferred to the mobile network by a direct route. The recommended model shows a more than $20 \%$ prediction error for the 100 -second prediction interval. Moreover, the model offloads $45 \%$ of mobile data for the applications with a delay threshold of 60 seconds.

Wang and $\mathrm{Wu}$ [11] recommended an offloading methodology that offloads mobile data and supports user requirement. The offloading methodology based on predicting the next access point, the automobile can come into contact with. This work assumes that the meeting interval of the cars and access points takes after the exponential Gaussian distribution. The car must compute the time to reach the next access point to make an offloading conclusion. If the approximated time is below the delay tolerance value, then the automobile must wait for the following access point for network connection. If the car never meets the succeeding access point as expected, then the car has to re-compute the meeting time by considering the prior computation and making a new offloading conclusion. However, if the approximated meeting time is higher than the delay tolerance value, then the vehicle will send the traffic to the mobile network by a direct route.

\section{RESULTS \& DISCUSSIONS}

\subsection{System Model}

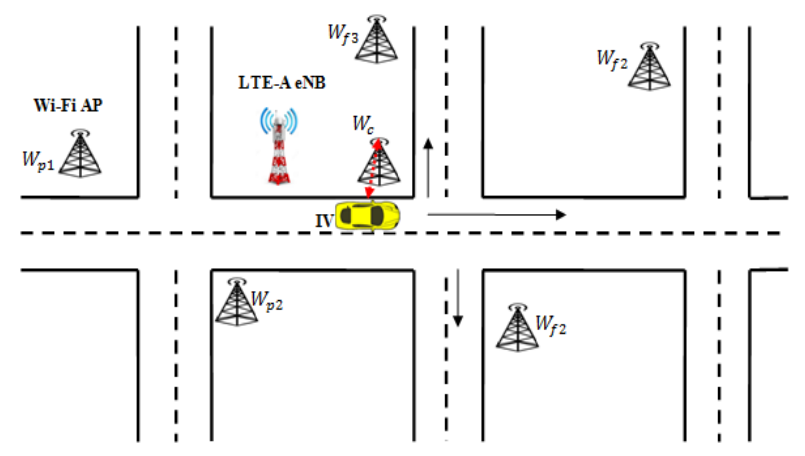

Figure 1. Opportunistic Offloading Scheme (OOS) System Model

HetVNET architecture is the basic framework of the system model for OOS methodology, as shown in Fig. 1. Manhattan model was used to set the actual scenario, and it is one of the essential mobility models for VANETs. It was made of vertical and horizontal streets with intersections. Each roadway has two lanes with opposite directions Vehicles can move forward, turn left or turn right at an intersection.

Wi-Fi network and 4G LTE-A network are two types of systems presently use. IEEE802.11 technology drives Wi-Fi network. From Fig. 1, the 4G LTE-A base station is called LTE-A eNB and IEEE802.11 access points are named as Wi-Fi APs. Each Wi-Fi access point is installed at the intersection of two roadways so that automobiles in both streets may achieve excellent communication without having radio signals pass through taller buildings. All Wi-Fi access points are in open access mode and has enough bandwidth to accommodate specific numbers of automobiles at the same time. Wi-Fi access points are not overlapping; they are placed at a certain distance apart. The OBU of the IVhas two network interfaces: one is to communicate with the mobile network, and the other is for Wi-Fi access point Each interesting has a Global Positioning System (GPS),

which is used to update the 
places where IV connect and disconnect to the specific WiFi access point.

\subsection{Prediction Criteria of Interest Vehicle}

We are using two criteria for predicting the future access point come into contact by IV, and these are such as historical data and vehicular trajectory. In historical details, the prediction table (PT) records the historical patterns of successful connected access points. This pattern consists of a prior access point $\left(W_{p}\right)$, present access point $\left(W_{c}\right)$, and future access point $\left(W_{f}\right)$. We compute two transition probabilities based on these three access points. First is the likelihood of the following access points $\left(W_{f 1}, W_{f 2} \ldots W_{f n}\right)$ using prior contacted access points $\left(W_{p 1}, W_{p 2 \ldots} W_{p n}\right)$. Second is and the probability of the future access point $\left(W_{f 1}, W_{f 2} \ldots W_{f n}\right)$ using the current connected access point $\left(W_{c}\right)$. Moreover, the vehicular trajectory is another essential criterion in determining if the IV is moving towards the predicted access point [12-14]. The vehicular path is computed whenever the IV needs to predict the succeeding access point. This will assure that the interest vehicle is moving towards the predicted access point. This automobile trajectory computation performs the derivation of the probability of the IV moving towards the succeeding access point $\left(W_{f 1}, W_{f 2} \ldots W_{f n}\right)$.

\subsection{Design of the Recommended Opportunistic Offloading Scheme}

When 4G LTE-A network is accessible and Received Signal Strength (RSS) from the access point $W_{c}$ is lower to the threshold $\left(T h_{r s s}\right)$ refer to Equation (1) for RSS computation. First, the IV has to compute the total number of transitions from $W_{c}$ to the succeeding $W_{f}$ using the data in prediction table (PT) as in Equation (2).

$R S S=P_{t}+G_{t}+G_{r}+L_{p}+L_{f}$

$H_{t}=\sum W_{f} \in P T\left(W_{c}\right)$

Where $P_{t}$ is the transmitted power, $G_{t}$ is the gain of transmitter, $G_{r}$ is the receiver gain, $L_{p}$ is the path loss and $L_{f}$ is the fading. The $H_{t}$ is the counting of the connection patterns in PT.

If $H_{t}$ has a higher value in comparison to the threshold ( $\alpha$ ) meaning, enough data is obtainable to predict the transition from $W_{c}$ to the succeeding $W_{f}$. The authors applied the first and second-order Markov chain concept to predict the change from $W_{c}$ to the succeeding $W_{f}$.

The IV starts to compute three probabilities using the data accumulated in PT and current IV moving direction. We compute these probabilities, as shown in Equation (3), Equation (4) and Equation (5).

A. Probability of the succeeding $\boldsymbol{W}_{\boldsymbol{f}}$ using prior contacted $\boldsymbol{W}_{\boldsymbol{p}}$

$\operatorname{Prob}_{1}=P\left(W_{c} \rightarrow W_{f} \mid W_{p}\right)=\frac{N\left(W_{c} \rightarrow W_{f} \mid W_{p}\right)}{N\left(W_{p} \rightarrow W_{c}\right)}$
Where $N\left(W_{c} \rightarrow W_{f} \mid W_{p}\right)$ is the number of times tuple $\left(W_{f}, W_{p}\right)$ has been noticed and $N\left(W_{p} \rightarrow W_{c}\right)$ is the sum of all rows with prior access point being $W_{p}$.

\section{B. Probability of the $\boldsymbol{W}_{\boldsymbol{f}}$ using current $\boldsymbol{W}_{\boldsymbol{c}}$}

$$
\operatorname{Prob}_{2}=P\left(W_{c} \rightarrow W_{f}\right)=\frac{N\left(W_{c} \rightarrow W_{f}\right)}{N\left(W_{c} \rightarrow W_{n}\right)}
$$

Where $N\left(W_{c} \rightarrow W_{f}\right)$ the counting of IV is moves from $W_{c}$ to $W_{f}$ and $N\left(W_{c} \rightarrow W_{n}\right)$ is the counting of the automobile moves from $W_{c}$ to any AP.

C. Probability of Interest Vehicle moves to the predicted $A P \boldsymbol{W}_{\boldsymbol{f}}$

$$
\operatorname{Prob}_{3}=\left(\frac{\pi-\tan ^{-1}\left(\frac{\sin (\theta+\beta)}{\cos (\theta+\beta)}\right)}{\pi}\right)
$$

where, $\mathrm{Prob}_{3}$ is the probability of IV moves to the predicted $\mathrm{AP} W_{f}, \theta$ is the angle between the IV position $C_{1}$ and succeeding $\operatorname{AP} W_{f}, \beta$ is the angle between automobile position $C_{2}$ and succeeding $\operatorname{AP} W_{f}$ at distance $d$ from $C_{1}$

After calculating all three probabilities of each row of the PT corresponding to the possible transitions, then the IV computes probability product of these three probabilities using Equation (6). The tuple with maximum $\operatorname{Prob}_{f}$ is the predicted $W_{f}$ the IV will access. Also, the IV computes the expected average bandwidth $\left(b_{f}\right)$ from $W_{f}$ using Equation (7). Furthermore, the IV estimates the time it will take to arrive at the succeeding AP using Equation (8).

$\operatorname{Prob}_{f}=\prod_{i=1}^{3} \operatorname{Prob}_{i}$

$b_{f}=\operatorname{Prob}_{f} \min (b)$

Where $\operatorname{Prob}_{f}$ is the probability product, $b_{f}$ is average bandwidth and $\mathrm{b}$ is the minimum bandwidth observed at $W_{f}$.

$t_{c f}=\frac{\sqrt{\left(x_{f}-x_{c}\right)^{2}-\left(y_{f}-y_{c}\right)^{2}}}{v_{c}}$

Where $t_{c f}$ is the approximated time required to reaching the predicted succeeding access point. The $v_{c}$ is the present speed of the interesting vehicle.

$C_{f}=b_{f}\left(t_{t h}-t_{c f}\right)$

$A_{f}=b_{f}\left(t_{t h}\right)$

Where $C_{f}$ is the data capacity that IV can acquire from the succeeding access point. The $A_{f}$ is the amount of the data the IV would transfer within the maximum tolerable delay (MTD). 
Let $t_{t h}$ is the MTD required for the particular application. Using $t_{c f}$, the IV can also predict the data capacity that it may acquire from the succeeding access point using Equation (9) and the amount of the data the IV would transfer within $t_{t h}$ by Equation (10) as in [16]. If $t_{c f}<t_{t h}$ and $C_{f}>A_{f}$ the IV delay its data transmission until it contacts with the succeeding access point. Therefore, the IV sends its information to the mobile network.

\section{PERFORMANCE COMPLEXITY OF OOS}

This section evaluates the time complexity of the recommended OOS. The time required to evaluate the OOS scheme based on the time for updating PT and calculating $C_{f}$, and $A_{f}$, which are represented by $T_{P T}$ and $T_{C A}$. To compute $C_{f}$ as in Equation (9), $b_{f}$, we compute $t_{c f}$, and $t_{t h}$ first. The complexity of computing $t_{t h}$ is constant since $t_{t h}$ is the maximum tolerable delay needed by the particular application. The computation of $b_{f}$ involves $\operatorname{Prob}_{f}$ which is the probability product of $\operatorname{Prob}_{1}, \operatorname{Prob}_{2}$ and $\operatorname{Prob}_{3}$, this shows linear computation complexity which depends on the size of PT. Therefore, the computation complexity of $b_{f}$ is $|O(P T)|$, where $|P T|$ denotes the size of PT. Likewise, the computation of $A_{f}$ requires computing $b_{f}$ which has the same computation complexity of $|O(P T)|$ as in $C_{f}$. As a result, the time complexity for computing $C_{f}$ and $A_{f}$ is $\left|O\left(T_{C A}\right)\right|=|O(P T)|+|O(P T)|=|O(P T)|$. Furthermore, the time complexity for updating the table PT is constant $T_{P T}$. This is the time spent on storing the update data of $W_{f}, N, b$ for the succeeding Wi-Fi AP. Therefore, the total time complexity of OOS scheme is given by $\left|O\left(T_{P T}\right)\right|+\left|O\left(T_{C A}\right)\right|=|O(1)|+|O(P T)|=|O(P T)|$ which indicates that it is only based on the size of PT.

\section{CONCLUSION}

Either to offload or not in HetVNET environment is very analytic, especially in supporting the QoS of various applications. The automobile needs to have previous data on the availability of the succeeding Wi-Fi access point and its expected capability. The paper recommended the OOS scheme, which assume the future Wi-Fi access point accessibility in a HetVNET environment. The explanatory design of the recommended MWAP scheme is described. Also, the prediction overhead of OOS scheme is analysed. The final analysis describes that the prediction overhead of OOS scheme mainly depends on the PT table. In the future, the recommended OOS will be benchmarked with related state-of-the-art systems. The comparison will focus on offloading ratio and prediction accuracy of the recommended OOS and the benchmarked systems.

\section{ACKNOWLEDGEMENTS}

This research was sponsored by the Ministry of Higher Education, Zanzibar University (ZU) and Universiti Kebangsaan Malaysia (UKM), under the Fundamental Research Grant Scheme (FRGS/1/2016/ICT03/UKM/02/2).

\section{REFERENCES}

1. C, Cisco. Visual Networking Index. Cisco Visual Networking Index: Global Mobile Data Traffic Forecast Update. pp. 2014-2019, 2017.

2. A. Aijaz, H. Aghvami, and M. Amani, "A survey on mobile data offloading: technical and business perspectives," Wireless Communications, IEEE, vol. 20, pp. 104-112, 2013.

3. F. Mobility, "Seamless Wireless Local Area Network (WLAN) Offload," ed: Stage, 2012.

4. G. Zhioua, M., H. Labiod, N. Tabbane, and S. Tabbane, "VANET Inherent Capacity for Offloading Wireless Cellular Infrastructure: An Analytical Study," in New Technologies, Mobility and Security (NTMS), 2014 6th International Conference on, 2014, pp. 1-5.

5. F. Malandrino, C. Casetti, C. Chiasserini, and M. Fiore, "Offloading cellular networks through ITS content download," in Sensor, Mesh and Ad Hoc Communications and Networks (SECON), 2012 9th Annual IEEE Communications Society Conference on, 2012, pp. 263-271.

6. M. Gramaglia, C. Bernardos, and M. Calderon, "Seamless internet $3 \mathrm{G}$ and opportunistic WLAN vehicular connectivity," EURASIP Journal on Wireless Communications and Networking, vol. 2011, p. 183, 2011.

7. M. François and G. Leduc, "AP and MN-Centric Mobility Prediction: A Comparative Study Based on Wireless Traces," in NETWORKING 2007. Ad Hoc and Sensor Networks, Wireless Networks, Next Generation Internet: 6th International IFIP-TC6 Networking Conference, Atlanta, GA, USA, May 14-18, 2007. Proceedings, I. F. Akyildiz, R. Sivakumar, E. Ekici, J. C. d. Oliveira, and J. McNair, Eds., ed Berlin, Heidelberg: Springer Berlin Heidelberg, 2007, pp. 322-332.

8. U. Rathnayake, M. Ott, and A. Seneviratne, "A DBN approach for network availability prediction," presented at the Proceedings of the 12th ACM international conference on Modeling, analysis and simulation of wireless and mobile systems, Tenerife, Canary Islands, Spain, 2009.

9. J. R. Pedrasa and A. P. Seneviratne, "MOBIX: System for managing mobility using information exchange," in Mobile and Ubiquitous Systems: Networking \& Services, MobiQuitous, 2009. MobiQuitous '09. 6th Annual International, 2009, pp. 1-10.

10. C. Sommer, S. Joerer, and F. Dressler, "On the applicability of Two-Ray path loss models for vehicular network simulation," in Vehicular Networking Conference (VNC), 2012 IEEE, 2012, pp. 64-69.

11. S. S. John, "Introduction to RF propagation," ed: WileyInterscience, 2005

12. Alawi, M. A., Alsaqour, R. A., Sundararajan, E., \& Ismail, M. Prediction model for offloading in vehicular Wi-Fi network. International Journal on Advanced Science, Engineering and Information Technology, 6(6), 944-951. 2016.

13. Alawi M, Alsaqour R, Ismail M, Sundararajan E, Abdelhaq M. Vehicular Wi-Fi Offloading in Heterogeneous Vehicular Networks: Techniques and Challenges. Mobile Networks and Applications.1-20, 2017.

14. Ahmad, R., Sundararajan, E. A., Othman, N. E., \& Ismail, M. Efficient Handover in LTE-A by Using Mobility Pattern History and User Trajectory Prediction. Arabian Journal for Science and Engineering. 43(6), 2995-3009, 2018. 